\title{
Functionalization layer influence on the Sensitivity of Surface Plasmon Resonance (SPR) biosensor
}

\author{
Dominique Barchiesi $^{\mathrm{a}}$, Nathalie Lidgi-Guigui ${ }^{\mathrm{b}}$, Marc Lamy de la Chapelle ${ }^{\mathrm{b}}$ \\ ${ }^{a}$ Project Group for Automatic Mesh Generation and Adaptation Methods \\ University of Technology of Troyes, France \\ 12 Rue Marie Curie - BP 2060, F-10010 Troyes Cedex, France \\ Tel: +33-(0)325718436; Fax: +33-(0)325715649 \\ ${ }^{b}$ CSPBAT - UMR CNRS 7244 - Group of Biomolecules Biological mediums \\ University of Paris 13 - UFR SMBH \\ 74 rue Marcel Cachin - 93017 Bobigny, France
}

\begin{abstract}
Plasmonic biosensors represent a rapidly expanding interdisciplinary field with numerous transducers which are based principally on the optical measurement of the plasmon shift, that is induced by the change of surrounding water optical index, due to the presence of biomaterials, in a binding event. A functionalization layer is practically used for selective detection of the molecules of interest. In this paper we show how this material can modify the sensitivity of the biosensor and we propose an optimization of the biosensor.

Keywords: Surface plasmon, Biosensor, Optimization.
\end{abstract}

\section{Introduction}

Among the large family of biosensors, those that are based upon the measurement of the perturbation of the Surface Plasmon Resonance, have an in-

Email address: dominique.barchiesi@utt.fr (Dominique Barchiesi) 
creasing expansion since the success in the engineering control of nanolayer fabrication that was first applied in 1983. Since, the Surface Plasmon Resonance (SPR) biosensor has become one of the most successful label-free sensor. The SPR technique is currently employed in biomolecular engineering, drug design, monoclonal antibody characterization, virus-protein interaction, environmental pollutants detection, among other interesting problems. A review of the various experimental setups and principles can be found in Refs. [1, 2, 3]. Surprisingly, the optimization of such structures $[4,5,6,7,8]$ and the influence of the functionalization layer have rarely been addressed. Therefore, the thickness of the gold layer is commonly chosen to $50 \mathrm{~nm}[9]$ or $55 \mathrm{~nm}[10]$ and neither the stick layer of Gold on glass nor the functionalization layer have been included in the models.

The sensing principle relies on the shift of the plasmonic resonance caused by the surrounding dielectric environmental change in a binding event. The basic principle of such transducer is the use of the existence of mathematical complex poles of the intensity of the reflected light, under particular illumination conditions (p-polarization), corresponding to a sudden absorbtion of light by the thin metallic layer, for a specific angle of incidence of the illumination varies $[11,7]$, leading to a highly sensitive device $[4,5]$. Kolomenskii et al proposed model and experiments that demonstrate that the measurement of the SPR shift is proportional to the concentration of the $\mathrm{NaCl}$ and ethanol. They also discussed the limit of detection according to the specifications of the detector and the source. Friebel and Meinke also proved the linear dependance of the optical index on the concentration of hemoglobin using Fresnel reflectance measurements [12]. Older studies focused on the re- 
fractive index of solutions at high concentrations and produced results with non linearities in dependance on concentration [13]. Conversely, using nanobiosensors, where the plasmon is localized due the geometry of nanoparticles, the normalized LSPR response has the same functional form as the Langmuir adsorption isotherm [14].

Nowadays, the basic principle of such commercial SPR biosensors is based on the measurement of the shift of the reflected intensity peak, which corresponds to the shift of the surface plasmon resonance induced by a change of optical index in the upper medium. This change of optical property is commonly related to the bending of biological molecules on a chemical functionalization of the metallic surface of the biosensor. Even in the case of the planar SPR biosensor, the problem of optimization is intrinsically NP-hard as it involves the inverse function of the reflected intensity, which is the square modulus of a transcendant function of complex variables. The sensitivity of the biosensor also depends on the contrast of the plasmon peak which optimization has been addressed without including the functionalization layer, with various numerical methods $[7,15,16,17]$.

In this paper, we will show the influence of the relative permittivity of a specific functionalization layer on the sensitivity of the SPR biosensor. Section 2 is devoted to the description of the model of the SPR biosensor and of the sensitivity. In Sec. 3 numerical results are presented and discussed, before concluding in Sec. 4. 


\section{The model of biosensor}

The SPR biosensor under investigation is made of two metal layers, which are deposited on a glass prism (Fig. 1). The relative permittivity of the glass prism is $\epsilon_{1}$. The relative permittivities $\epsilon_{2}$ and $\epsilon_{3}$ of the chromium and gold layers are complex numbers and depend on the incoming wavelength $\lambda_{0}$ which is fixed in this study to $670 \mathrm{~nm}$. We suppose that the variations of permittivities related to the small thickness of materials and the mode of deposition is not a critical parameter [18]. The thicknesses of chromium ans gold layer are $e_{1}$ and $e_{2}$. The functionalization is an homogenous medium of thickness $e_{3}$ and relative permittivity $\epsilon_{4}$. This parameter differs from relative permittivity of the medium of detection is $\epsilon_{5}$ and its variations could influence the design of the metal layers, with the aim of improving the sensitivity of the biosensor. In the following, the modification of $\epsilon_{5}$ due to the presence or the absence of biological molecules is supposed to be $\Delta \epsilon_{5}$. The roughness of the gold layer is nanometric and will be neglected in this study. The angle of incidence of the incoming, monochromatic, p-polarized plane wave is $\theta$. For a particular parameters set $\left(\theta, e_{1}, e_{2}\right)$, the reflected intensity $R$ vanishes, sign of the energy transfer of the incoming light to the thin metal layer (excitation of a plasmon resonance). 


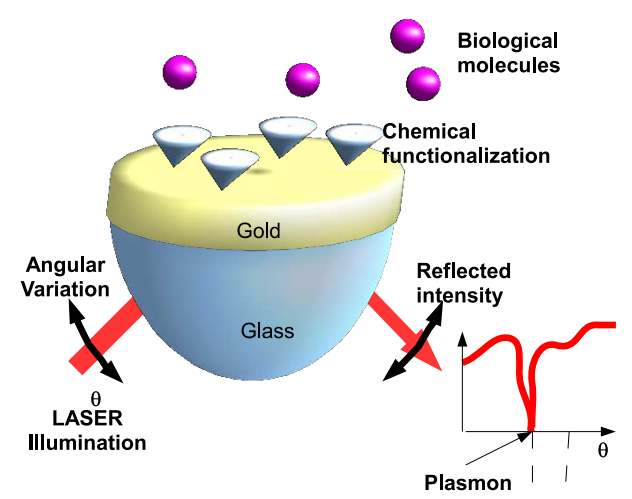

(a)

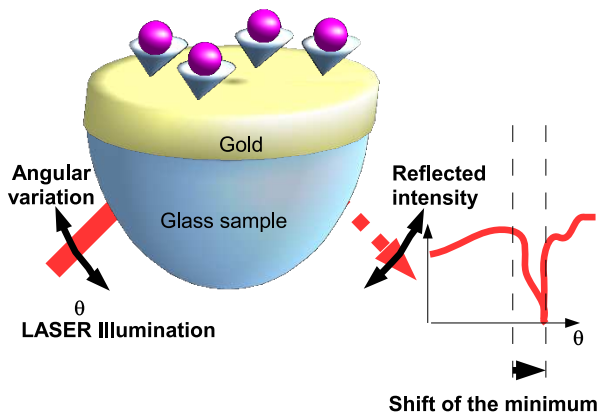

(b)

Figure 1: Schematic of the biosensor used to measure variations of the optical index of the above medium as a function of biomaterials concentration.

The detection being based on the measurement of the angular shift of the minimum of reflectivity, the sensitivity can be defined as a function of the shift:

$$
\Delta \theta=\theta\left(\min \left(R\left(\theta, \epsilon_{5}+\Delta \epsilon_{5}\right)\right)\right)-\theta\left(\min \left(R\left(\theta, \epsilon_{5}-\Delta \epsilon_{5}\right)\right)\right)
$$

The maximum of $\Delta \theta$ is searched as a function of the metal thicknesses $e_{1}$ and $e_{2}$. This function is implicit and therefore requires numerical inversion. The well known Nelder-Mead method is used in this study.

On the other hand, the quality of the resonance depends on the reflected intensity $R(\theta)$ and is characterized by the shape and the minimum of this function. The shape depends on the relative permittivity of materials. The minimum of $R(\theta)$ must be as small as possible to get the higher transfer of the incoming energy to the metal layers. Therefore, the visibility $V$ of $R(\theta)$ is also a guarantee of the quality of measurements $[19,4,10]$ : 


$$
V=\frac{\max (R)-\min (R)}{\max (R)+\min (R)}
$$

Therefore, the sensitivity $S$ of the SPR biosensor can be therefore deduced, in order to take into account both $V$ and $\Delta \theta$ :

$$
S=S\left(e_{1}, e_{2}\right)=\frac{V}{\Delta \theta}
$$

The calculation of the reflected intensity $R=\left|r_{15}\right|^{2}$ uses the Fourier transform of the Maxwell partial differential equations, to generate a linear system between the amplitudes of the plane waves in each medium, the boundary conditions at infinity to select the wave vectors and the continuity of the tangential magnetic field and the normal component of the electric excitation $[20,21,7]$. The reflected amplitude of field is proportional to $r_{15}$ which can be deduced after some basic algebra :

$$
r_{15}\left(\epsilon_{i}, e_{i}, \theta\right)=\frac{N}{D}
$$

with:

$$
\begin{aligned}
N= & e^{2 \imath e_{2}\left(w_{3}+w_{4}\right)} r_{2} r_{3} r_{4}+e^{2 \imath\left(e_{1} w_{2}+e_{2}\left(w_{3}+w_{4}\right)\right)} r_{1} r_{3} r_{4}+e^{2 \imath\left(e_{1}\left(w_{2}+w_{3}\right)+e_{3} w_{4}\right)} r_{1} r_{3} r_{3}+ \\
& e^{2 \imath\left(e_{1} w_{2}+e_{2} w_{4}\right)} r_{4}+e^{2 \imath\left(e_{1} w_{3}+e_{3} w_{4}\right)} r_{3}+e^{2 \imath\left(e_{2} w_{3}+e_{3} w_{4}\right)} r_{2}
\end{aligned}
$$

$+e^{2 \imath\left(e_{1} w_{2}+e_{2} w_{3}+e_{3} w_{4}\right)} r_{1}$

and:

$$
\begin{aligned}
D= & \left(r_{2} e^{2 \imath e_{1} w_{2}}+r_{1}\right)\left(e^{2 \imath\left(e_{1} w_{3}+e_{2} w_{4}\right)} r_{4}+e^{2 \imath\left(e_{1} w_{3}+e_{3} w_{4}\right)} r_{3}\right)+ \\
& \left(r_{2} r_{1}+e^{2 \imath e_{1} w_{2}}\right)\left(e^{2 \imath\left(e_{2}\left(w_{3}+w_{4}\right)\right)} r_{3} r_{4}\right)+e^{2 \imath\left(e_{2} w_{3}+e_{3} w_{4}\right)},
\end{aligned}
$$


with $\imath=\sqrt{-1}$ and $r_{i}$ the Fresnel's reflection coefficient on the interface separating two mediums of relative permittivities $\epsilon_{i}$ and $\epsilon_{i+1}$ respectively:

$$
r_{i}=\frac{\epsilon_{i+1} w_{i}-\epsilon_{i} w_{i+1}}{\epsilon_{i+1} w_{i}+\epsilon_{i} w_{i+1}}
$$

and $w_{i}=\frac{2 \pi}{\lambda_{0}} \sqrt{\epsilon_{i}-\sin ^{2}(\theta)}$, the $z$ component of the wave vector in medium of permittivity $\epsilon_{i}$ (Fig. 1).

The result in Eq. 4 has been verified by considering mediums with equal permittivities to get the simple Fresnel coefficients, and only one non null thickness to recover the coefficient of reflection by single slab. Moreover, it has been checked with Eq. (40) of Ref. [22].

As shown in [15], both reflected intensity $R$ and $\theta_{S P}=\theta(\min (R))$ depend strongly on the metal thicknesses $e_{1}$ and $e_{2}$. The purpose of the following section is to give insights and numerical results on the sensitivity of the biosensor.

\section{Numerical study of the sensitivity of the SPR biosensor}

The goal is to determine thicknesses $e_{1}$ and $e_{2}$ for the best sensitivity $S$. The starting values for the Nelder-Mead algorithm are those of experiments [10] and previous optimizations, without the functionalization layer [7]. Two modes of detection are considered: in water $\left(\epsilon_{5}=1,7689\right)$ and in air $\epsilon_{5}=1$ (dry mode). The target variation of the relative permittivity of the above medium is $\Delta \epsilon_{5}=0.001$. This variation is due to the presence of the molecules to be detected. The relative permittivity of the glass prism is $\epsilon_{1}=2.25$. The relative permittivities $\epsilon_{2}=-1.75+20.6 \imath$ and $\epsilon_{3}=-11.9+1.1 \imath$ 
of the chromium and gold layers are complex numbers at the incoming wavelength $\lambda_{0}=670 \mathrm{~nm}$. The functionalization is an homogenous medium of thickness $e_{3}=5 \mathrm{~nm}$ and relative permittivity $\epsilon_{4}=1.96$. This value has been measured by ellipsometry for a monolayer of Keyhole Limpet Hemocyanin (KLH), deposited on a $50 \mathrm{~nm}$ thick gold slab. This molecule is typical of many functionalization layers in terms of optical properties.

In this study, the optimization of the two parameters $e_{1}$ and $e_{2}$ is made with Nelder-Mead algorithm. Nevertheless, we have verified the results that could depend on the starting value, and are not constrained in a limited physical domain of variations, by using heuristic methods described in Refs. $[7,15,16,17]$. The four investigated cases are combination of with/without functionalization and detection in water or air. In the absence of functionalization the relative permittivity of the corresponding layer is $\epsilon_{4}=\epsilon_{5}$. An equivalent way consists in considering $e_{3}=0$.

The figures 2 and 3 shows exemples of maps of the visibility $V$ (Eq. 2), the shift $\Delta \theta$ of the plasmon resonance (Eq. 1), the sensitivity $S$ (Eq. 3) and the minimum of $R(\theta)$. Figures 2 differs from Figs. 3 because the conditions of plasmon excitation vary in water and air. The contrast of permittivities $\epsilon_{5} / \epsilon_{1}$ and therefore the critical angles [20] are not the same. Moreover, the sensitivity $S$ is twice in water than that in air (c). Nevertheless, the plasmon shift is greater if the detection is made in water (b). This difference is mainly due to the behavior of the visibility $V$ (a). The figure 4 illustrates the influence of the functionalization layer on both sensitivity as defined above, and the shift of plasmon resonance, for both modes of detection. The computation have been made for $\Delta \epsilon_{5}=0.001$, that corresponds to a variation of the above op- 
tical index equal to $3 \cdot 10^{-2}$. The shift decay in water is greater than in air but remains small compared to the uncertainty on angular measurements. The sensitivity is also slightly modified. Nevertheless, both exhibit flat maximum around the commonly used set of thickness $\left(e_{1} \approx 50 \mathrm{~nm}, e_{2} \approx 2 \mathrm{~nm}\right)$.

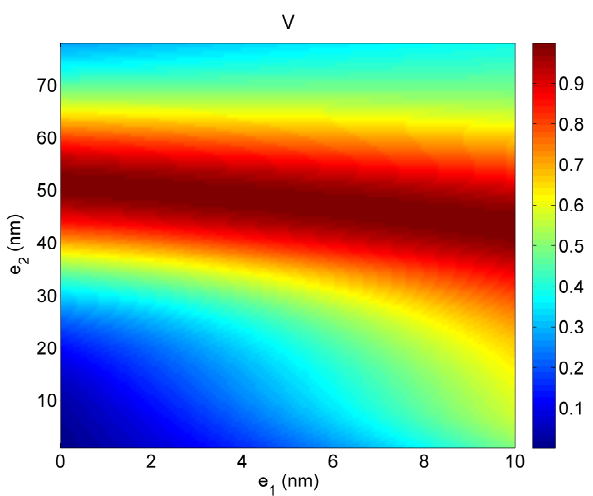

(a)

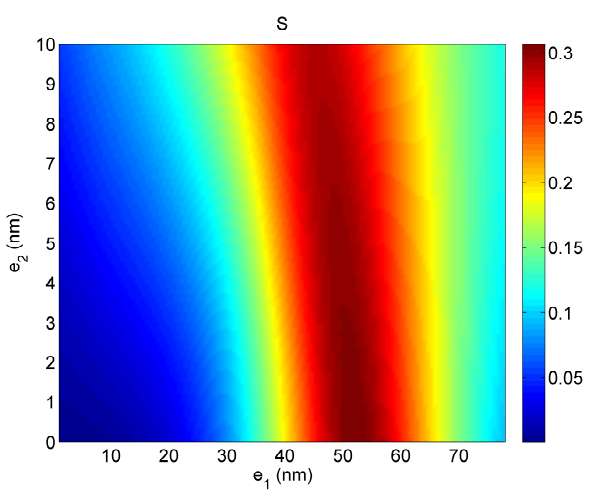

(c)

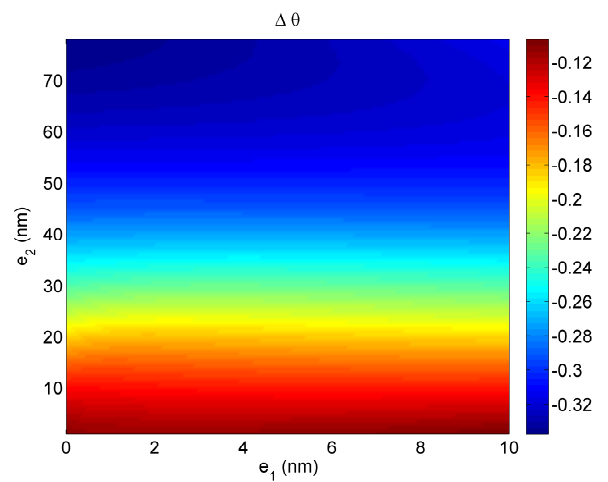

(b)

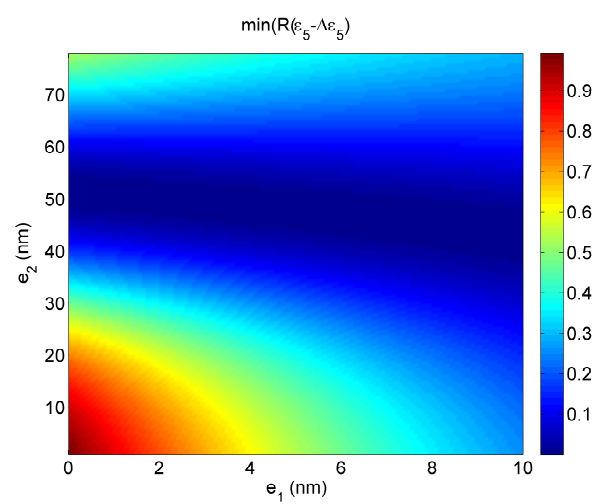

(d)

Figure 2: Characteristic of the three layers SPR biosensor as a function of metal thicknesses $e_{1}$ and $e_{2}$. The detection is in water. The functionalization layer is included. (a) Visibility $V\left(e_{1}, e_{2}\right)$, (b) Shift of SPR $\Delta \theta\left(e_{1}, e_{2}\right)$, (c) Sensitivity $S\left(e_{1}, e_{2}\right),(\mathrm{d}) \min (R)\left(e_{1}, e_{2}\right)$. 


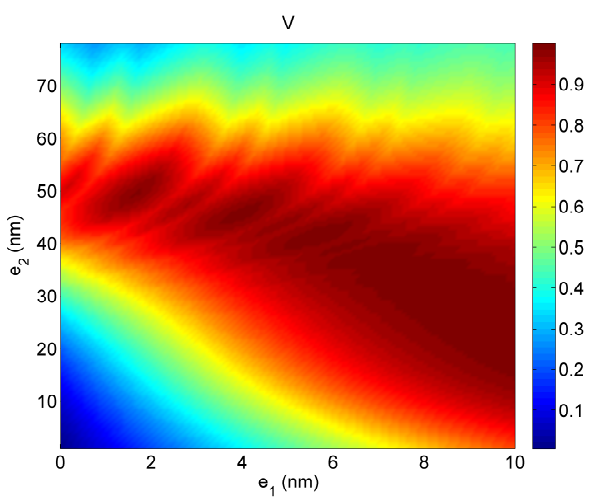

(a)

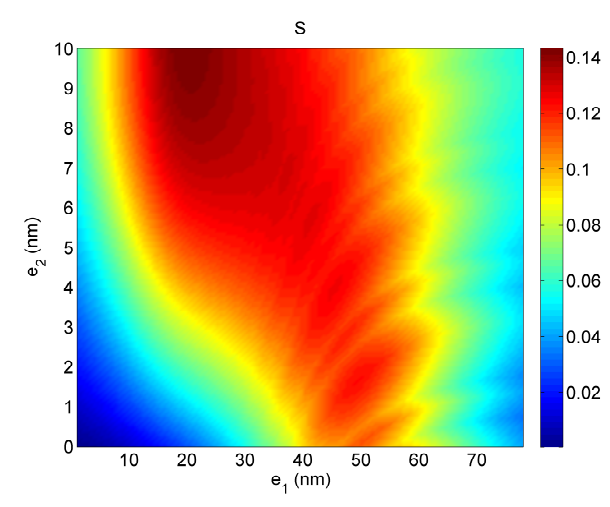

(c)

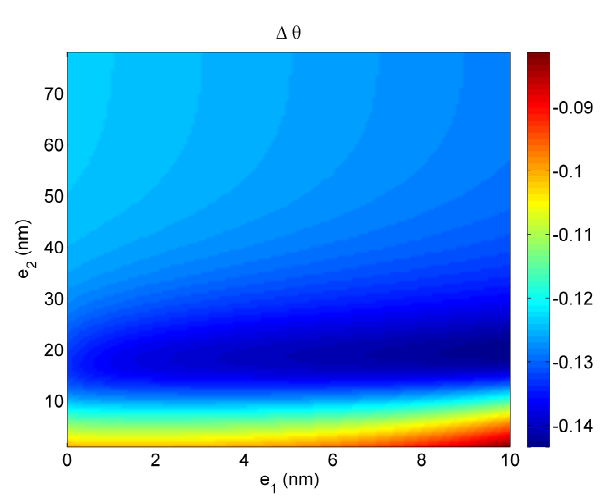

(b)

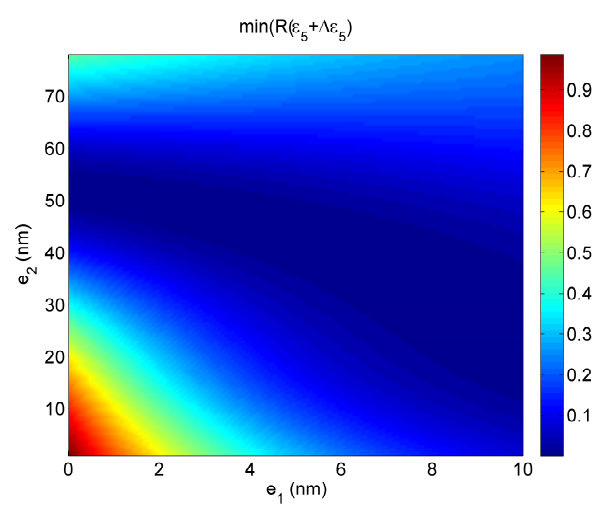

(d)

Figure 3: Characteristic of the three layers SPR biosensor as a function of metal thicknesses $e_{1}$ and $e_{2}$. The detection is in air. The functionalization layer is included. (a) Visibility $V\left(e_{1}, e_{2}\right)$, (b) Shift of SPR $\Delta \theta\left(e_{1}, e_{2}\right)$, (c) Sensitivity $S\left(e_{1}, e_{2}\right),(\mathrm{d}) \min (R)\left(e_{1}, e_{2}\right)$. 


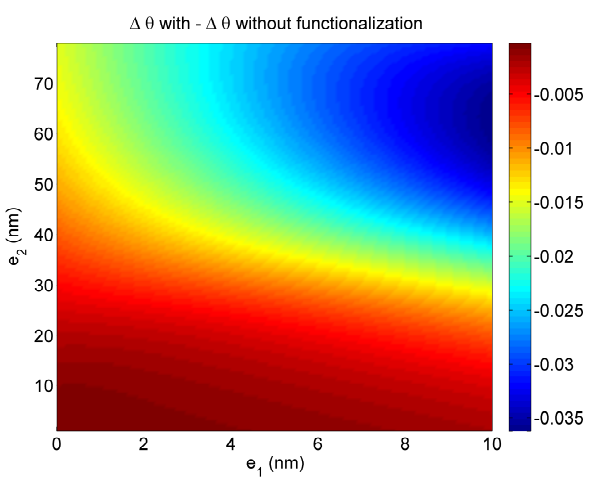

(a)

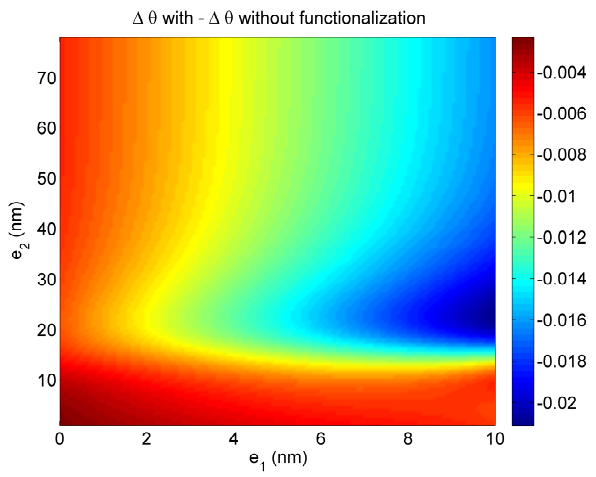

(c)

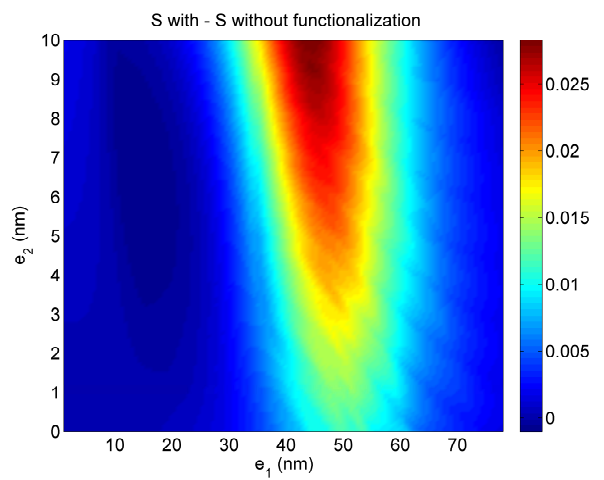

(b)

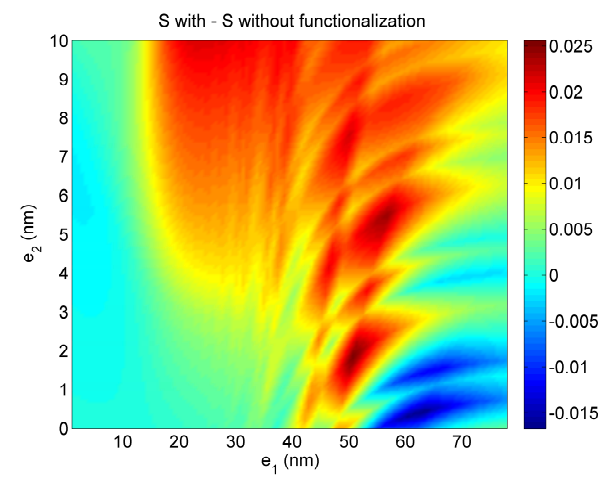

(d)

Figure 4: Influence of the functionalization layer. Difference of the shift of SPR $\Delta \theta\left(e_{1}, e_{2}\right)$ in water (a), in air (c). Difference of the sensitivity $S$ in water (b), in air (d).

Therefore, for the illuminating wavelength $\lambda_{0}=670 \mathrm{~nm}$, the numerical optimization of the thicknesses deserves to be computed. The table 1 gives the optimized parameters obtained from the Nelder-Mead method. As expected by the visual inspection of Fig.2, 3 and of course 4, the best set of thicknesses is not the same if the detection is made in water or in air. Moreover, surprisingly, the tiny difference of the results obtained with inclusion of the functionalization layer lead to an non negligible difference of thicknesses. 
Some conclusions could be drawn from these results. The sensitivity of the biosensor is better in air than in water. Actually, the plasmon resonance is more efficient if $\epsilon_{5} / \epsilon_{1}$ is smaller. The thickness of chromium should be as small as possible, the chromium being highly dispersive at these wavelength. Nevertheless, the relative permittivity of the functionalization layer is slightly different from that of water and more for air and even if the thickness $e_{3}$ is only $5 \mathrm{~nm}$ and located more than $50 \mathrm{~nm}$ away from the glass/Cr interface, it influences on the sensitivity. The optimal thickness of gold is more than 2 $\mathrm{nm}$ less in the case of detection in air than in water, but the functionalization layer modify these values of less than $1 \mathrm{~nm}$. Of course, this variation could be increased if the thickness of the functionalization layer is increased.

\begin{tabular}{cccccc}
\cline { 2 - 5 } & \multicolumn{2}{c|}{ Water } & \multicolumn{2}{c|}{ Air } \\
& With F & Without F & With F & Without F \\
\hline$e_{1}(\mathrm{~nm})$ & 1.2 & 1.0 & 1.6 & 1.0 \\
$e_{2}(\mathrm{~nm})$ & 51.7 & 52.4 & 49.7 & 49.1 \\
$S$ & 3.3 & 3.5 & 8.1 & 16.8
\end{tabular}

Table 1: Optimization of the metal thicknesses $e_{1}$ and $e_{2}$, with or without functionalization, with detection in air or in water.

\section{Conclusion}

In this study, the sensitivity of the SPR biosensor has been defined as a function of the quality of the plasmon resonance and of its shift, to describe the method of measurement. The numerical results show that the sensitivity depends on the thin functionalization layer, the detection being made either 
in water or in air. Moreover, the thicknesses of both the chromium stick layer and the gold layer have been optimized for the two modes of detection. The sensitivity of the SPR biosensor should be also determined, for example by using the method described in Ref [18] to determine if the tolerance on the thickness falls within the nanometric differences mentioned above. Moreover, the nanometric roughnesses could be introduced, but it would require a more expensive model and an experimental evaluation of the RMS of the gold layer.

\section{acknowledgement}

Author thank the Région Champagne-Ardennes, the Conseil Régional de l'Aube and the European Project (FP7 Health-F5-2009-241818) for financial supports.

[1] X. Hoaa, A. Kirk, M. Tabrizian, Towards integrated and sensitive surface plasmon resonance biosensors: A review of recent progress, Biosensors and Bioelectronics 23 (2007) 151-160.

[2] B. Liedberg, C. Nylander, I. Lundström, Bionsensing with surface plasmon resonance - how it all started, Biosensors \& Biolectronics 10 (1995) $1-9$.

[3] J. Homola, S. S. Yee, G. Gauglitz, Surface plasmon resonance sensors: Review, Sensors and Actuators B 54 (1999) 3-15.

[4] A. Kolomenskii, P. Gershon, H. Schuessler, Sensitivity and detection limit of concentration and absorption measurements by laser-induced surface-plasmon resonance, Appl. Opt. 36 (1997) 6539-6547. 
[5] P. Lecaruyer, E. Maillart, M. Canva, J. Rolland, Generalization of the rouard method to an absorbing thin-film stack and application to surface plasmon resonance, Appl. Opt. 45 (2006) 8419-8423.

[6] S. Ekgasit, C. Thammacharoen, F. Yu, W. Knoll, Influence of the metal film thickness on the sensitivity of surface plasmon resonance biosensors, Appl. Spectroscopy 59 (2005) 661-667.

[7] D. Barchiesi, D. Macías, L. Belmar-Letellier, D. Van Labeke, M. Lamy de la Chapelle, T. Toury, E. Kremer, L. Moreau, T. Grosges, Plasmonics: Influence of the intermediate (or stick) layer on the efficiency of sensors, Appl. Phys. B 93 (2008) 177-181.

[8] J. Homola, On the sensitivity of surface plasmon resonance sensors with spectral interrogation, Sensors and Actuators B 41 (1997) 207-211.

[9] D. R. Shankarana, K. V. Gobia, T. Sakaic, K. Matsumotoc, K. Tokod, N. Miuraa, Surface plasmon resonance immunosensor for highly sensitive detection of 2,4,6-trinitrotoluene, Biosensors and Bioelectronics 20 (2005) 1750-1756.

[10] H. Neff, W. Zong, A. Lima, M. Borre, G. Holzhüter, Optical properties and instrumental performance of thin gold films near the surface plasmon resonance, Thin Solid Films 496 (2006) 688-697.

[11] E. Kretschmann, The ATR method with focused light - application to guided waves on a grating, Optics Communications 23 (1) (1978) 41-44.

[12] M. Friebel, M. Meinke, Model function to calculate the refractive index 
of native hemoglobin in the wavelength range of 250-1100 nm dependent concentration, Appl. Opt. 45 (2006) 2838-2842.

[13] W. M. B. M. Yunus, A. B. A. Rahman, Refractive index of solutions at high concentrations, Appl. Opt. 27 (1988) 3341-3343.

[14] J. C. Riboh, A. J. Haes, A. D. McFarland, C. R. Yonzon, R. V. Duyne, A nanoscale optical biosensor: Real-time immunoassay in physiological buffer enabled by improved nanoparticle adhesion, J. Phys. Chem. B 107 (2003) 1772-1780.

[15] D. Barchiesi, E. Kremer, V. Mai, T. Grosges, A Poincaré's approach for plasmonics: The plasmon localization, J. Microscopy 229 (2008) 525532 .

[16] D. Barchiesi, Adaptive non-uniform, hyper-ellitist evolutionary method for the optimization of plasmonic biosensors, in: Proc. Int. Conf. Computers \& Industrial Engineering CIE 2009, 2009, pp. 542-547. doi:10.1109/ICCIE.2009.5223795.

[17] S. Kessentini, D. Barchiesi, T. Grosges, L. Giraud-Moreau, M. L. de la Chapelle, Adaptive non-uniform particle swarm application to plasmonic design, International Journal of Applied Metaheuristic Computing (IJAMC) 2 (1) (2011) 18-28.

[18] T. Grosges, D. Barchiesi, S. Kessentini, G. Gréhan, M. Lamy de la Chapelle, Nanoshells for photothermal therapy: A monte-carlo based numerical study of their design toler- 
ance, Biomedical Optics Express 2 (6) (2011) 1584-1596, http://www . opticsinfobase. org/boe/abstract.cfm?URI=boe-2-6-1584.

[19] E. M. Yeatman, Resolution and sensitivity in surface plasmon microscopy and sensing, Biosensors \& Bioelectronics 11 (6-7) (1996) 635649 .

[20] M. Born, E. Wolf, Principle of Optics, Pergamon Press, Oxford, 1993.

[21] D. Barchiesi, A 3-D multilayer model of scattering by nanostructures. application to the optimisation of thin coated Nano-Sources, Optics Communications 126 (1996) 7-13.

[22] J. M. Vigoureux, Polynomial formulation of reflection and transmission by stratified planar structures, J. Opt. Soc. Am. A 8 (11) (1991) 16971701. 